\title{
Modelo de Matriz: ferramenta para a construção de boas práticas em saúde mental comunitária
}

\section{Matrix Model: a tool for the construction of good practice in community mental health}

\author{
Elisabete Ferreira Mângia ${ }^{1}$, Melissa Tieko Muramoto ${ }^{2}$
}

\begin{abstract}
MÂNGIA, E. F.; MURAMOTO, M. T. Modelo de Matriz: ferramenta para a construção de boas práticas em saúde mental comunitária. Rev. Ter. Ocup. Univ. São Paulo, v. 20, n. 2, p. 118-125, maio/ago. 2009.
\end{abstract}

RESUMO: As mudanças contemporâneas produzidas pelas novas políticas de saúde mental colocam como desafio central a construção de serviços substitutivos ao hospital, desenvolvidos no contexto da atenção na comunidade. Tal desafio exige um complexo conjunto de condições que implicam, dentre outros fatores, no desenvolvimento de uma nova cultura ética e técnica capaz de criar respostas inovadoras para atenção às pessoas com transtornos mentais. É nesse cenário que se deve discutir a pertinência e a importância do projeto elaborado por Thornicroft e Tansella no "Modelo de Matriz" que é apresentado neste artigo. Nele os autores trazem contribuições fundamentais para alicerçar a nova cultura de serviços e formação profissional pretendidas pelo processo de reforma psiquiátrica. Este modelo pode ser utilizado no planejamento, avaliação e modelagem dos serviços além de trazer instrumentos úteis para a pesquisa em serviços de saúde mental.

DESCRITORES: Serviços comunitários de saúde mental/tendências e planejamento. Política de Saúde Mental.

1. Prof ${ }^{\mathrm{a}} \mathrm{Dr}^{\mathrm{a}}$ do Curso de Terapia Ocupacional da FMUSP.

2. Terapeuta ocupacional, mestre em Ciências da Reabilitação, Técnica do Curso de Terapia Ocupacional da FMUSP.

Endereço para correspondência: Departamento de Fisioterapia, Fonoaudiologia e Terapia Ocupacional da FMUSP. Rua Cipotânea, 51, Cidade Universitária, São Paulo, SP, CEP 05360-160. 


\section{INTRODUÇÃO}

$\mathrm{N}$ a atualidade, as mudanças produzidas pelas novas políticas de saúde mental colocam como desafio central a construção de serviços substitutivos ao hospital, desenvolvidos no contexto da atenção na comunidade.

Saraceno (2000), ao discutir o panorama e características comuns dos processos de reforma psiquiátrica nos países europeus, aponta que os anos 1980 foram marcados pelo debate em torno aos tratamentos e suas possíveis especificidades para cada diagnóstico. Tal movimento ocorreu em paralelo ao avanço agressivo das abordagens farmacológicas, o que, em larga medida, resultou em estratégias simplificadoras diante da magnitude e complexidade do problema representado pelos transtornos mentais.

Já nos anos 1990, se pôde perceber um importante deslocamento de foco e a crescente percepção sobre a importância do papel dos serviços de saúde mental. Nesse sentido o autor advoga que, na atualidade, não se pode mais discutir tratamentos fora do contexto dos serviços onde eles acontecem. (SARACENO, 2000).

Para ele "não há clínica sem serviço", o que significa a compreensão de que, por serviços, não se deve entender apenas o conjunto de edifícios ocupados por equipes assistenciais, mas o "complexo conjunto de políticas, estratégias, planejamento e ações organizativas", ou seja, "não há clínica sem um conjunto racional de serviços" (SARACENO, 2000).

O desenvolvimento de redes de serviços substitutivos, adequados, acessíveis e empenhados em garantirem a continuidade da assistência, passou a ser considerado o mais importante e, ao mesmo tempo, talvez, o mais difícil desafio das reformas psiquiátricas. Exige um complexo conjunto de condições, que inclui a capacidade de desenvolver respostas adequadas às necessidades diversificadas dos usuários, além da ampliação da oferta a grupos ainda marginalizados da assistência (OMS, 2001; THORNICROFT; TANSELLA, 2008a)

Apesar dos inegáveis avanços, estão ainda presentes nesse campo as resistências sociais e políticas que impedem o apoio mais amplo aos processos de desinstitucionalização e a criação e sustentação das redes de serviços substitutivos (OMS, 2001; SARACENO, 1999).

Acredita-se que a dificuldade no desenvolvimento de uma nova cultura técnica, organizativa e ética, e não apenas a insuficiência numérica e a falta de recursos dos serviços, contribuem significativamente ao abandono de pessoas com transtornos mentais severos e ao retorno às práticas de internação e asilamento (MERCIER; FLEURY, 2002).

Agrava esse quadro a falta de respostas adequadas ao projeto da saúde mental comunitária no âmbito da formação dos profissionais de saúde, que ainda são hegemonicamente preparados para as práticas tradicionais, centradas em procedimentos e distantes da cultura do trabalho em equipe.

Nessa perspectiva, a depender do projeto e forma de organização, os novos serviços protagonizam o risco de reproduzir a lógica e resultados institucionais que num primeiro momento visavam combater, assim a mudança do repertório dos técnicos de saúde mental e a necessária inovação das práticas desenvolvidas pelos serviços se colocam como fatores determinantes para o êxito do projeto da desinstitucionalização (SARACENO, 1999; MÂNGIA; MURAMOTO, 2006).

Estudos recentes confirmam que, em quase todos os países que vem desenvolvendo políticas de saúde mental ancoradas nas linhas comuns disseminadas pela Organização Mundial de Saúde, dentre eles o Brasil, a organização de novos serviços enfrenta barreiras semelhantes e coloca em cena, dentre outros aspectos, a dificuldade da maior parte dos países em desenvolverem e sustentarem tais políticas. Nesse cenário, se deve levar em conta a dificuldade em priorizar o desenvolvimento de serviços substitutivos; a presença ainda forte dos hospitais psiquiátricos, a falta de conhecimento sobre as necessidades de saúde mental da população e a dificuldade em definir prioridades assistenciais desse campo (THORNICROFT; TANSELLA, 2008a; THORNICROFT; TANSELLA, LAW, 2008).

As dificuldades relacionadas à formulação do objetivo dos serviços, sua modelagem e critérios para avaliação e aprimoramento parecem jogar um papel importante nesse contexto.

Estudos nacionais recentes que buscam avaliar o processo de implantação dos Centros de Atenção Psicossociais demonstram a necessidade de construir instrumentos que confiram aos serviços e suas equipes capacidade de desenvolver práticas coerentes com os princípios e desafios colocados pela Política Nacional de Saúde Mental. Apesar dos evidentes avanços na organização das redes de serviços de saúde mental, nesses estudos foram observadas carências importantes, não apenas estruturais e de financiamento, mas na formação e suporte para o trabalho técnico e na modelagem e gestão dos serviços que comprometem a qualidade, sustentabilidade e reprodutibilidade do novo modelo assistencial (CAMPOS, et al., 2009; SILVA et al., 2009; JARDIM et al., 2009).

Há muitas evidências que configuram o que alguns autores denominam de crise de operatividade dos serviços, 
que no geral, tendem a não responderem satisfatoriamente as demandas e necessidades dos usuários, apresentam uma organização fragmentada em procedimentos e competências das diversas corporações profissionais que os compõem, não desenvolvem ações territoriais, nem definem claramente prioridades assistenciais e níveis de responsabilidade sobre a população sob seus cuidados. São identificadas práticas desnecessárias, institucionalizantes e desprovidas de sentido para os usuários, além do abandono de pessoas com transtornos muito graves cujo manejo se mostra difícil. O recurso a internação e a trans-institucionalização muitas vezes pode ser compreendido como um dos sintomas dessa crise (MÂNGIA; MURAMOTO, 2006).

Tais avaliações também apontam para a dificuldade de criação de uma linguagem comum e compartilhada entre todos os atores que compõe as redes de serviços. Faltam, sobretudo, definição e compartilhamento de critérios e indicadores que possam subsidiar a caracterização e mensuração dos objetivos e resultados pretendidos e contribuir no processo de avaliação das novas redes e serviços.

Enfim, o campo da saúde mental requer ainda o desenvolvimento e aprimoramento de uma nova cultura técnica e ética e é no contexto das redes de serviços que se tem a possibilidade desse desenvolvimento. Assim, a discussão em torno do papel, recursos e modelagem dos serviços se tornam central e dela depende o sucesso no avanço e consolidação do novo modelo.

É nesse cenário que se deve discutir a pertinência e a importância do projeto elaborado por Thornicroft e Tansella no "Modelo de Matriz". Nele, os autores além de formularem com clareza e precisão o campo da Saúde Mental Comunitária, apresentam e defendem a importância de um mapa que possa auxiliar na formulação de metas e passos necessários para a implementação de serviços e também para o diagnóstico das suas disfuncionalidades. (THORNICROFT; TANSELLA, 2008).

Tais proposições são desenvolvidas no contexto de um quadro de referência construído a partir da articulação concomitante de 3 níveis: o nível nacional/regional (no qual são formuladas as políticas, por exemplo), o local (onde devem estar localizados os serviços comunitários) e o individual (no qual há maior necessidade de desenvolvermos ações e práticas baseadas em princípios éticos, evidências e experiências).

No desenvolvimento e aplicação do modelo são considerados também 3 eixos indispensáveis e indissociáveis, são eles: 1 . O princípio ético; 2 . As práticas baseadas em evidências e 3. O acúmulo de experiências (THORNICROFT; TANSELLA, 2000).
Os autores apontam quatro objetivos a serem alcançados com a utilização do modelo:

1) Oferecer um modelo que facilite a descrição dos serviços de saúde mental; que possa simplificar a descrição da estrutura e função dos serviços e permitir criar bases para a avaliação comparada dessas estruturas complexas, possibilitando a construção de uma linguagem comum que possa gerar intercâmbios e compreensão compartilhada por parte de todos os atores envolvidos. Os autores reconhecem os problemas decorrentes da simplificação presente em qualquer modelo, e por isso buscam um equilíbrio entre simplicidade e especificidade adequada e necessária em sua formulação;

2) Oferecer um modo de coordenar eventos complexos que se verificam em períodos diversos, ao introduzir critérios de ordenamento que se acoplam a um modelo bidimensional, cujas dimensões são tempo e espaço. Tais dimensões, vistas como independentes, ao serem consideradas em interação oferecem uma vasta gama de dimensões úteis para representar a variabilidade encontrada nos serviços de saúde mental.

Dessa forma, o modelo se coloca como um instrumento integrativo que se adapta aos serviços de saúde mental, caracterizados por processos desagregadores tais como: as dificuldades de gestão e financiamento; a fragilidade de manutenção e continuidade das experiências; a dificuldade de fixação e formação dos recursos humanos; a diversidade de linguagens e posturas ideológicas e teóricas, frente à questão das doenças mentais, seu diagnóstico e formas de tratamento; os conflitos entre equipes e psiquiatras - que parece ser um fenômeno internacional descrito por muitos autores - que geram ações contrastantes no contexto de um mesmo serviço.

Os autores discutem um aspecto que tem sido negligenciado nos estudos e propostas de construção de serviços de saúde mental. A natureza do trabalho com pessoas com transtornos mentais, em especial os transtornos severos, traz aos trabalhadores e serviços diversas exigências e uma sobrecarga específica, que merece atenção e cuidado, pois também geram forças desestabilizadoras que podem contribuir para o aparecimento da síndrome do Burnout. Em tal contexto é importante pensar um método que contribua para a coerência e coesão de processos de trabalho assim complexos. (THORNICROFT; TANSELLA, 2000).

3) Favorecer a compreensão desses eventos e, enfim, facilitar a identificação das carências dos serviços. Para compreender o funcionamento dos serviços, o modelo se detém em explicar eventos de relevância clínica em relação ao seu deslocamento espaço - temporal, isto é, nas dimensões de "onde" e "quando", que podem ajudar a 
compreender o "como" e o "porquê".

4) Identificar e induzir o estabelecimento das ações prioritárias para a melhoria dos serviços. Nesse aspectos os autores defendem a importância de um guia operativo, salientam a necessidade de realizar o diagnóstico, a definição de estratégias e as ações prioritárias para compor as intervenções.

O objetivo maior do modelo é que ele ajude a todos os atores envolvidos, profissionais, administradores, usuários dos serviços, etc. a utilizarem, como ponto de partida para suas ações, um conhecimento mais articulado do próprio serviço em seu contexto, de forma a definir ações específicas e singulares dotadas de sentido para tal contexto. Isso poderia contribuir para o desenvolvimento de padrões de qualidade adequados aos serviços de saúde mental de um novo tipo, a exemplo dos padrões adotados em outros setores da saúde. No geral, há um contraste entre os padrões de qualidade adotados em outros serviços sanitários e a falta de padrões para a saúde mental (THORNICROFT; TANSELLA, 2000).

\section{O princípio ético como principal eixo de orientação para a melhoria dos serviços de saúde mental}

Ao definirem os três eixos orientadores do Modelo de Matriz (ética, evidências e experiências), Thornicroft e Tansella (2008) definem que estes devem ser considerados de forma indissociável, ou seja, não é possível considerar uma intervenção que seja efetiva, porém antiética, ou qualquer estratégia ética, mas que não esteja pautada em evidências ou experiências já compartilhadas. Além disso, definem que tais eixos devam ser considerados de forma hierárquica, e colocam a ética em primeiro lugar, fazendo a defesa de que os princípios éticos devem ser construídos e deixados claros já na fase de planejamento dos serviços. Quando tais princípios não são compartilhados abertamente, os conflitos de valor podem aparecer durante a prática e dificultar ou mesmo inviabilizar projetos coletivos (THORNICROFT; TANSELLA, 2008).

Nesta perspectiva, o Modelo de Matriz dialoga perfeitamente com as proposições disseminadas pela OMS, no que diz respeito a importância do estabelecimento de princípios que possam orientar a implementação dos serviços e a prática clínica dos profissionais. O eixo ético, proposto no Modelo de Matriz, pode ser considerado complementar aos princípios e ingredientes da atenção para a organização de serviços de saúde mental, definidos pela OMS (2001, 2003)

Assim, de acordo com a hierarquia proposta, os autores discutem os princípios éticos em 3 níveis: nacional, local e individual.

No nível nacional, é discutida a necessidade da existência de leis a acordos nacionais e internacionais, que garantam o exercício e o gozo de direitos humanos básicos pelas pessoas com transtornos mentais. Sobre este aspecto os autores afirmam que

"podemos estimar, com precisão, o valor atribuído às pessoas com transtornos mentais apenas a partir da quantidade de atenção dirigida a assegurar que elas sejam tratadas de maneira completamente humanizada" (THORNICROFT; TANSELLA, 2008, p.32).

Nesse sentido, a Declaração dos Direitos Humanos, da Organização das Nações Unidas - ONU, é considerada o principal código de defesa dos direitos humanos, além das Convenções Internacionais de Direitos Civis e Políticos e de Direitos Econômicos, Sociais e Culturais. Tais acordos se aplicam a todos os países que os ratificaram (OMS, 2001; THORNICROFT; TANSELLA, 2008).

Como desdobramento, em 1991, foram adotados os Princípios para a Proteção de Pessoas Acometidas por Transtorno Mental e para a Melhoria da Assistência à Saúde Mental (ONU), que estabelecem os direitos e liberdades básicas que devem ser assegurados a essa população. Apesar de não ter validade jurídica tal como as Convenções anteriormente citadas, este documento é considerado importante, pois reúne um conjunto de direitos básicos invioláveis das pessoas com transtornos mentais, estejam elas em tratamento ou não (OMS, 2001). A importância dessa questão levou alguns países a adotarem convenções e acordos próprios em relação às pessoas com transtornos mentais. É o caso do Conselho Europeu, que reúne 43 países da Europa, e da Organização Panamericana de Saúde, através da Declaração de Caracas (OMS, 2001; THORNICROFT; TANSELLA, 2008).

Apesar do reconhecimento sobre a importância destes acordos e de sua utilidade, adotados pela OMS em seus manuais e relatórios, Thornicroft e Tansella (2008) problematizam a inexistência de um consenso internacional que determine os princípios básicos que devem guiar a construção e organização de serviços e, especificamente, o cuidado em saúde mental. Assinalam ainda a importância de se considerar a definição de princípios específicos para um contexto e momento histórico determinado, apontando como exemplos os casos da África do Sul, que construiu toda a Política de Saúde Mental pós-apartheid, e o da própria Inglaterra, que incorporou os princípios de diversos acordos internacionais na construção de seu Plano Nacional de Saúde Mental (THORNICROFT; TANSELLA, 2008).

Observa-se também a proximidade do modelo de matriz com os guias lançados pela OMS a partir de 2003. 
São ao todo 14 guias de saúde mental que contemplam temas inter-relacionados para o planejamento e desenvolvimento de políticas e serviços de saúde mental. Por exemplo, o guia referente à Organização dos Serviços de Saúde Mental (OMS, 2003) apresenta princípios e diretrizes, que podem ser complementadas e operacionalizadas por meio das proposições do Modelo de Matriz para o nível local.

Se comparamos as proposições sugeridas pela OMS com as do Modelo de Matriz identificamos grande identidade entre ambas. Os princípios estabelecidos pela OMS são: acessibilidade, compreensividade, coordenação e continuidade do cuidado, efetividade, equidade e respeito aos direitos humanos. O modelo de matriz considera os seguintes princípios: autonomia, continuidade, efetividade, acessibilidade, compreensividade, equidade, responsabilidade, coordenação e eficiência.

De acordo com a OMS, o princípio da acessibilidade deve garantir a disponibilidade de serviços de saúde mental locais, evitando-se que as pessoas tenham que viajar longas distâncias ou por muitas horas. A acessibilidade aos serviços é um fator importante para promover a continuidade do cuidado e para garantir que todas as pessoas possam ter acesso ao cuidado quando e onde precisarem. O Modelo de Matriz complementa esse principio ao afirmar que este também se aplica ao arranjo interno dos serviços e não apenas à sua localização.

Um arranjo de serviço acessível permite que as pessoas não tenham que passar por esperas longas e burocráticas para avaliação e início de seu tratamento. O princípio da acessibilidade também deve garantir acesso a todas as pessoas que necessitem dos serviços, sem barreiras seletivas a grupos determinados (por diagnósticos, raça, grau de severidade ou quaisquer outros), além da disponibilidade em oferecer atendimento durante a noite e aos finais de semana (OMS, 2003; THORNICROFT; TANSELLA, 2008).

Já o princípio da compreensividade pode ser considerado em duas dimensões. Por compreensividade horizontal se define a extensão atingida pelo serviço no que diz respeito a sua capacidade de promover cuidado entre os diversos graus de severidade dos transtornos mentais e diversas características das pessoas (idade, gênero, diagnóstico). Por compreensividade vertical, se define a capacidade que a rede de serviços tem de incluir todos os elementos que sejam necessários para o enfrentamento das necessidades e demandas complexas das pessoas com transtornos mentais. Dessa forma, o conjunto exato de serviços dependerá do contexto em que a rede está inserida, considerando fatores sociais, econômicos e culturais (OMS, 2003; THORNICROFT; TANSELLA, 2008).

Os princípios de coordenação e continuidade do cuidado podem ser compreendidos conjuntamente. Por coordenação entende-se a característica do serviço em desenvolver ações de forma coordenada, não apenas entre os profissionais do mesmo serviço, mas também entre os diversos serviços que possam ser acessados pelas pessoas atendidas, considerando-se a complexidade de suas demandas e necessidades (OMS, 2003). Para Thornicroft e Tansella (2008), a coordenação também implica na exigência de haver planos de tratamento para cada usuário e destes terem "metas claras e incluir intervenções que sejam necessárias e efetivas: nem mais, nem menos." (p.38). O princípio de continuidade do cuidado pode ser compreendido enquanto a capacidade do serviço em desenvolver ações nos níveis local e individual que sejam coerentes entre si e no decorrer do tempo, entre diferentes profissionais e equipes.

O princípio de efetividade implica na necessidade dos serviços e profissionais desenvolverem ações efetivas, orientadas por evidências (OMS, 2003). Para o Modelo de Matriz, a efetividade deve ser compreendida em termos dos benefícios intencionais e comprovados, para o contexto real de vida das pessoas, que os serviços são capazes de oferecer (THORNICROFT; TANSELLA, 2008).

O princípio de equidade deve ser compreendido enquanto possibilidade de garantir que o acesso das pessoas com transtornos mentais aos serviços seja baseado em necessidades. A equidade deve ser enfatizada durante o planejamento e avaliação dos serviços, especialmente na fase de estabelecimento das prioridades. Além disso, é necessário considerar que as pessoas com transtornos mentais graves, geralmente com maiores necessidades, são tendencialmente menos capazes de apresentarem demandas aos serviços. Assim, a distribuição dos recursos oferecidos pelos serviços deve ser baseada em critérios de equidade (OMS, 2003; THORNICROFT; TANSELLA, 2008).

O último princípio estabelecido pela OMS é o de respeito aos direitos humanos, que implica em reconhecimento e empoderamento das pessoas com transtornos mentais, estímulo a autonomia para tomada de decisões que afetam suas vidas e uso de tipos de tratamento menos restritivos possíveis. (OMS, 2003) Este princípio é abordado em profundidade pelo Modelo de Matriz no nível nacional.

Além dos princípios apresentados acima, Thornicroft e Tansella elegem os princípios de autonomia, que consiste na capacidade das pessoas em tomar decisões e fazer escolhas de forma independente, promovida de forma direta por meio de intervenções efetivas da equipe de saúde mental; o princípio de responsabilidade, que implica na garantia de que todas as pessoas envolvidas no cuidado: 
profissionais, usuários e familiares, serviços e público em geral, tenham expectativas legítimas em relação a como os serviços devem agir com responsabilidade; e o princípio da eficiência, que consiste na característica do serviço de maximizar os resultados a partir de um nível específico de ações iniciais.

No nível individual, além das garantias previstas pelas Declarações e Convenções Internacionais, os autores do Modelo de Matriz apontam para a importância da utilização de técnicas de tratamento menos restritivas possíveis, e para a escolha por tratamentos e intervenções que sejam fortemente apoiados pelas evidências disponíveis.

O principal objetivo do estabelecimento de princípios nos três níveis, nacional, local e individual, é garantir que soluções técnicas ou visões de grupos orientados por ideais heterogêneos não sejam usados separadamente de uma base sólida de princípios éticos, para orientar a escolha $\mathrm{e}$ o planejamento do melhor cuidado para as pessoas com transtornos mentais. O que os autores sugerem é que haja um modelo de triangulação, que permita considerar necessariamente a ética, as melhores práticas baseadas em evidências e o acúmulo de experiências (OMS, 2001, 2003; THORNICROFT; TANSELLA, 2008).

A partir desta orientação, Thornicroft e Tansella apresentam o Modelo de Matriz e propõem que este possa atuar como um norteador para as práticas de cuidado em saúde mental.

\section{O Modelo de Matriz: dimensões geográfica e temporal}

O Modelo de Matriz é composto por duas dimensões: geográfica e temporal (Figura I). A dimensão geográfica foi inserida, pois os autores acreditam e defendem que os serviços devam ser organizados prioritariamente em seus contextos locais, de forma que sejam acessíveis para aqueles que precisam. Esta dimensão divide-se em três níveis: nacional/regional, local e individual.

FIGURA 1 - O Modelo de Matriz

\begin{tabular}{l|c|c|c}
\hline & \multicolumn{2}{|c|}{ Dimensão Temporal } \\
\hline Dimensão geográfica & (A) Fase de Entrada & (B) Fase de processo & (C) Fase de resultados \\
\hline (1) Nível nacional/regional & $1 \mathrm{~A}$ & $1 \mathrm{~B}$ & $1 \mathrm{C}$ \\
\hline (2) Nível local & $2 \mathrm{~A}$ & 2B & 2C \\
\hline (3) Nível individual & $3 \mathrm{~A}$ & $3 \mathrm{~B}$ & $3 \mathrm{C}$ \\
\hline
\end{tabular}

Thornicroft, Tansella, 2008

O nível nacional/regional é importante por ser o nível no qual a política de saúde mental é formulada e as leis são estabelecidas, bem como a definição da alocação de recursos e das prioridades de implantação dos serviços. Para este nível, é importante que sejam definidos e monitorados padrões mínimos de cuidado para a população, e a atualização dos currículos profissionais de acordo com os avanços clínicos e de pesquisas da área.

O nível local é compreendido como uma "lente" que possibilita ver com maior clareza a efetividade das leis e políticas de saúde mental em vigência no país. A organização deste nível dependerá em grande medida da forma de organização utilizada em cada país, ou seja, se os serviços serão organizados em distritos ou setores de saúde (Inglaterra e EUA), por áreas geográficas ou comunidades e ainda por área de cobertura populacional. O ponto relevante deste nível é a afirmação da necessidade dos serviços serem organizados localmente, de forma a oferecer cuidado na comunidade, isto é, próximo a casa das pessoas (THORNICROFT; TANSELLA, 2008).
O nível individual corresponde ao cuidado oferecido diretamente à pessoa com transtorno mental, seus familiares e rede social próxima. É geralmente compreendido como o território exclusivo do profissional de saúde, mas os autores defendem que os resultados individuais dependem fortemente do que é definido nos níveis nacional e local. A questão de maior enfoque neste nível é a necessidade das práticas e intervenções serem baseadas em evidências, já que o que se observa com maior freqüência é um distanciamento entre o sugerido pelas evidências e a prática clínica real.

A segunda dimensão organizadora é a temporal, na qual é possível identificar um fluxo de eventos que ocorre desde o princípio da intervenção até os resultados. Neste fluxo, os autores consideram que a fase dos resultados é a mais importante e que os serviços devem ser avaliados pelos resultados que produzem. A fase temporal também é composta por três fases: fase de entrada, fase de processos e fase de resultados.

A fase de entrada caracteriza-se pelos recursos introduzidos na rede de serviços de saúde mental, que podem 
ser separados entre entradas visíveis, que são os recursos físicos como prédios e profissionais, e entradas invisíveis, definidas, por exemplo, pelas relações de trabalho e políticas de saúde mental. A fase de processo é definida pelas atividades (clínicas e não-clínicas) desenvolvidas durante a oferta do cuidado. Esta fase é considerada importante, pois aqui serão estabelecidas as prioridades e as estratégias para o desenvolvimento do cuidado, que levarão aos resultados da intervenção. A fase dos resultados define a eficiência e efetividade das intervenções propostas pelo serviço. A avaliação dos resultados precisa ser garantida e deve acontecer de forma acurada e rotineira, como prática geral da equipe, uma vez que o objetivo principal dos serviços é melhorar os resultados para as pessoas com transtornos mentais e para o sistema de saúde mental em geral.

\section{Considerações finais}

A intenção de apresentar, mesmo que em linhas gerais, o Modelo de Matriz decorre da constatação de sua importância para o atual cenário nacional de organização dos serviços e formação de profissionais no campo da saúde mental. Concordamos com a proposição dos autores quando advogam que este modelo pode ser utilizado no planejamento, avaliação e modelagem dos serviços além de trazer instrumentos úteis para a pesquisa em serviços de saúde mental.

Os motivos do reconhecimento internacional dos trabalhos de Graham Thornicroft e Michele Tansella, que já são consenso entre pesquisadores do campo e diversas agencias nacionais e internacionais de saúde, podem se tornar agora acessível aos profissionais e usuários.

Mais do que um modelo, o último livro publicado por esses autores, que será em breve traduzido para o português, traz aprofundamento teórico importante que consolida e qualifica o campo da saúde mental comunitária como o único capaz de gerar respostas adequadas aos problemas enfrentados pelas pessoas com transtornos mentais.

Dessa forma, sua utilização deve ser recomendada e estimulada, pois ele vem sendo considerado um importante divisor de águas na literatura atual sobre a organização de serviços de saúde mental.

MÂNGIA, E. F.; MURAMOTO, M. T. Matrix Model: a tool for the construction of good practice in community mental health. Rev. Ter. Ocup. Univ. São Paulo, v. 20, n. 2, p.118-125, maio/ago. 2009.

\begin{abstract}
The contemporary changes produced by the new mental health policies place as main challenge the construction of substitutive services to hospital, developed in the context of care in the community. This challenge requires a complex set of conditions involving, among other factors, the development of a new ethical and technical culture able to create innovative responses to care of people with mental disorders. It is in this scenario that we must discuss the relevance and importance of the project developed by Thornicroft and Tansella, "The Matrix Model", presented in this paper. There, the authors bring fundamental contributions to underpin the new culture of services and professional training sought by the process of psychiatric reform. This model can be used in planning, evaluation and modeling of services; in addition, it can bring useful tools to the research in mental health services.
\end{abstract}

KEY WORDS: Community mental health services/trends and planning. Mental health policy/ Trends.

\section{REFERÊNCIAS}

CAMPO, R. T. O.; FURTADO, J. P.; PASSOS, E.; FERRER, A. L.; MIRANDA, L.; GAMA, C. A. P. Avaliação da rede de centros de atenção psicossocial: entre a saúde coletiva e a saúde mental. Rev. Saúde Pública, v. 43, Supl. 1, p. 16-22, 2009.

JARDIM, V.M.R.; CARTANA, M.H.F.; KANTORSKI, L.P.; QUEVEDO, A.L.A. Avaliação da política de saúde mental a partir dos projetos terapêuticos de Centros de Atenção Psicossocial. Texto Contexto Enf., v. 18, n. 2, p. 241-248, 2009.
MÂNGIA, E. F.; MURAMOTO, M. T. Integralidade e a construção de novas profissionalidades no contexto dos serviços substitutivos de saúde mental. Rev. Ter. Ocup. USP, v. 17, n. 3, 2006.

MERCIER C.; FLEURY, M. J. Integrated local networks as a model for organizing mental health services, Admin. Policy Mental Health, v. 30, n. 1, 2002.

OMS. Relatório sobre a saúde no mundo. Saúde mental: nova 
MÂngia, E. F.; MURAmoto, M. T. Modelo de Matriz. Rev. Ter. Ocup. Univ. São Paulo, v. 20, n. 2, p. 118-125, maio/ago. 2009.

concepção, Nova Esperança. 2001.

SARACENO, B. Libertando identidades. Da reabilitação psicossocial à cidadania possível. Belo Horizonte: Instituto Franco Basaglia/TeCorá, 1999.

SARACENO, B. Presentazione dell'edizione italiana, In; THORNICROFT, G., TANSELLA, M. Manuale per la riforma dei servizi di salute mentale. Um modello a matrice. Roma: Il Pensiero Cientifico Editore, 2000

SILVA, M. T., LANCMAN, S.; ALONSO, C. M. C. Conseqüências da intangibilidade na gestão dos novos serviços de saúde mental. Rev. Saúde Pública, v. 43, Supl. 1, p. 36-42, 2009.

THORNICROFT, G.; TANSELLA, M. Better mental health care. Londres: Cambridge University Press, 2008.
THORNICROFT, G.; TANSELLA, M. Quais são os argumentos a favor da atenção comunitária à saúde mental? Pesq. Práticas Psicossociais, v. 3, n. 1, p. 9-25, 2008a.

THORNICROFT, G.; TANSELLA, M. Manuale per La riforma dei servizi di salute mentale. Um modello a matrice. Roma: Il Pensiero Cientifico Editore, 2000

THORNICROFT, G.; TANSELlA, M.; LAW, A. Steps, challenges and lessons in developing community mental health care. World Psychiatry, v. 7, p. 87-92, 2008.

WHO. Organization of Services For Mental Health. Mental Health Policy and Service Guidance Package. 2003. Available from: http://www.who.int/mental_health/resources/en/Organization. pdf 